\title{
Article \\ Investigation of the k-Analogue of Gauss Hypergeometric Functions Constructed by the Hadamard Product
}

\author{
Mohamed Abdalla ${ }^{1,2, *(D)}$ and Muajebah Hidan ${ }^{1}$ (D) \\ 1 Department of Mathematics, Faculty of Science, King Khalid University, Abha 61471, Saudi Arabia; \\ mhedan@kku.edu.sa \\ 2 Department of Mathematics, Faculty of Science, South Valley University, Qena 83523, Egypt \\ * Correspondence: moabdalla@kku.edu.sa
}

check for updates

Citation: Abdalla, M.; Hidan, M. Investigation of the k-Analogue of Gauss Hypergeometric Functions Constructed by the Hadamard Product. Symmetry 2021, 13, 714. https://doi.org/10.3390/sym13040714

Academic Editor: Dmitry V. Dolgy and Paolo Emilio Ricci

Received: 15 March 2021

Accepted: 15 April 2021

Published: 18 April 2021

Publisher's Note: MDPI stays neutral with regard to jurisdictional claims in published maps and institutional affiliations.

Copyright: (c) 2021 by the authors. Licensee MDPI, Basel, Switzerland. This article is an open access article distributed under the terms and conditions of the Creative Commons Attribution (CC BY) license (https:// creativecommons.org/licenses/by/ $4.0 /)$.

\begin{abstract}
Traditionally, the special function theory has many applications in various areas of mathematical physics, economics, statistics, engineering, and many other branches of science. Inspired by certain recent extensions of the k-analogue of gamma, the Pochhammer symbol, and hypergeometric functions, this work is devoted to the study of the k-analogue of Gauss hypergeometric functions by the Hadamard product. We give a definition of the Hadamard product of k-Gauss hypergeometric functions (HPkGHF) associated with the fourth numerator and two denominator parameters. In addition, convergence properties are derived from this function. We also discuss interesting properties such as derivative formulae, integral representations, and integral transforms including beta transform and Laplace transform. Furthermore, we investigate some contiguous function relations and differential equations connecting the HPkGHF. The current results are more general than previous ones. Moreover, the proposed results are useful in the theory of k-special functions where the hypergeometric function naturally occurs.
\end{abstract}

Keywords: Hadamard product; generalized hypergeometric functions; integral representations; integral transforms; differential equations

MSC: 33B15; 33C20; 33C90; 33E20

\section{Introduction}

In recent years, the hypergeometric function of parameters consisting of two numerators and one denominator has become of increasing significance due to its applications in many fields of biophysics, quantum mechanics, spectroscopy, engineering, and other scientific areas (see, e.g., [1-4] and the references therein).

Recently, numerous attempts have been made to generalize and improve on hypergeometric function by using an extension of the Pochhammer symbol. The reader may consult, for example, the recent works of Srivastava et al. [5,6], Jana et al. [7,8], Agarwal et al. [9,10], Fuli et al. [11], Hidan et al. [12,13] and Abdalla [14-17].

One class of extensions of hypergeometric functions comprises the so-called khypergeometric functions in terms of the k-analogue of gamma and the Pochhammer symbol, which can be found in the work of Diaz and Pariguan [18].

Nowadays, some mathematical properties, such as integrals' formulae, inequalities, differential equations, contiguous function relations, generating functions and fractional calculus related to the k-hypergeometric function of parameters consisting of two numerators and one denominator have been presented in many references, for instance, Mubeen et al. [19-22], Rahman et al. [23], Chinra et al. [24], Korkmaz-Duzgun and ErkusDuman [25], Nisar et al. [26], Li and Dong [27], Kiryakova [28], Naz and Naeem [28] and Yilmazer and Ali [29]. 
In addition, the Hadamard product of two hypergeometric functions has been widely used in factorizing a newborn function, which is generalization as a hypergeometric series (see, [30,31]).

Inspired by the definition of a k-hypergeometric function [18], the authors introduce a new expansion of the k-analogue of Gauss hypergeometric functions by using the Hadamard product, which is the so-called Hadamard product of k-Gauss hypergeometric functions (HPkGHF) associated with the fourth numerator and two denominator parameters. The manuscript is organized as follows. In Section 2, we introduce the Hadamard product of k-Gauss hypergeometric functions and discuss their regions of convergence as well as the differentiation formulae. In Section 3, we obtain some integral representations and integral transforms including a beta transform, a Laplace transform, and an inverse Laplace transform of the Hadamard product of k-Gauss hypergeometric functions. In addition, contiguous function relations and differential equations connecting the HPkGHF are established in Section 4. Finally, in Section 5, we reach the conclusion remarks.

Throughout our present work, $\mathbb{N}:=\{1,2,3, \ldots\}$ denotes the set of natural numbers, $\mathbb{N}_{0}=\mathbb{N} \cup\{0\}, \mathbb{Z}^{-}:=\{-1,-2,-3, \ldots\}$ denotes the set of negative integers, $\mathbb{Z}_{0}^{-}=\mathbb{Z}^{-} \cup\{0\}$, $\mathbb{R}^{+}$denotes the set of positive real numbers and $\mathbb{C}$ denotes the set of complex numbers.

\section{Preliminaries}

The hypergeometric function known also as a Gauss function is defined by

$$
G\left(\delta_{1}, \delta_{2}, \delta_{3} ; u\right)=\sum_{n=0}^{\infty} \frac{\left(\delta_{1}\right)_{n}\left(\delta_{2}\right)_{n}}{\left(\delta_{3}\right)_{n}} \frac{u^{n}}{n !}, u \in \mathbb{C}
$$

which is absolutely and uniformly convergent if $|u|<1$, divergent when $|u|>1$, and is absolutely convergent when $|u|=1$, if $\operatorname{Re}\left(\delta_{3}-\delta_{1}-\delta_{2}\right)>0$, where $\delta_{1}, \delta_{2}, \delta_{3}$ are complex parameters with $\delta_{3} \in \mathbb{C} \backslash \mathbb{Z}_{0}^{-}$,

$$
\left(\delta_{1}\right)_{n}=\frac{\Gamma\left(\delta_{1}+n\right)}{\Gamma\left(\delta_{1}\right)}= \begin{cases}\delta_{1}\left(\delta_{1}+1\right) \ldots\left(\delta_{1}+n-1\right), & n \in \mathbb{N}, \delta_{1} \in \mathbb{C} \\ 1, & n=0 ; \delta_{1} \in \mathbb{C} \backslash\{0\},\end{cases}
$$

is the Pochhammer symbol (or the shifted factorial), and $\Gamma($.$) is gamma function.$

Diaz and Pariguan [18] defined the k-analogue of gamma, beta and hypergeometric functions as follows.

Definition 1. For $k \in \mathbb{R}^{+}$, the $k$-gamma function $\Gamma^{k}(u)$ is defined by:

$$
\Gamma^{k}(u)=\int_{0}^{\infty} y^{u-1} e^{-\frac{u^{k}}{k}} d y=\lim _{m \rightarrow \infty} \frac{m ! k^{m}(m k)^{\frac{u}{k}-1}}{(u)_{m, k}}
$$

where $u \in \mathbb{C} \backslash k \mathbb{Z}^{-}$. We note that $\Gamma^{k}(u) \rightarrow \Gamma(u)$, for $k \rightarrow 1$, where $\Gamma(u)$ is the classical Euler's gamma function and $(u)_{m, k}$ is the $k$-Pochhammer symbol given by:

$(u)_{m, k}=\frac{\Gamma^{k}(u+m k)}{\Gamma^{k}(u)}= \begin{cases}u(u+k) \ldots(u+(m-1) k), & m \in \mathbb{N}, u \in \mathbb{C} \\ 1, & m=0, k \in \mathbb{R}^{+}, u \in \mathbb{C} \backslash\{0\} ;\end{cases}$

the relation between the $\Gamma^{k}(u)$ and the gamma function $\Gamma(u)$ follows easily that:

$$
\Gamma^{k}(u)=k^{\frac{u}{k}-1} \Gamma\left(\frac{u}{k}\right) .
$$


Definition 2. For $u, v \in \mathbb{C}$ and $k \in \mathbb{R}^{+}$, the $k$-beta function $\mathbf{B}^{k}(u, v)$ is defined by:

$$
\mathbf{B}^{k}(u, v)=\frac{1}{k} \int_{0}^{1} y^{\frac{u}{k}-1}(1-y)^{1-\frac{v}{k}} d y=\frac{\Gamma^{k}(u) \Gamma^{k}(v)}{\Gamma^{k}(u+v)},
$$

where $\operatorname{Re}(u)>0$ and $\operatorname{Re}(v)>0$.

Clearly, the case $k=1$ in (5) reduces to the known beta function $\mathcal{B}(u, v)$, and the relation between the k-beta function $\mathbf{B}^{k}(u, v)$ and the original beta function $\mathcal{B}(u, v)$ is:

$$
\mathbf{B}^{k}(u, v)=\frac{1}{k} \mathcal{B}\left(\frac{u}{k}, \frac{v}{k}\right) .
$$

Definition 3. Let $k \in \mathbb{R}^{+}$and $s_{1}, s_{2}, \eta \in \mathbb{C}$ and $s_{3} \in \mathbb{C} \backslash \mathbb{Z}_{0}^{-}$, then the $k$-Gauss hypergeometric function is defined in:

$$
{ }_{2} \digamma_{1}^{k}\left[\begin{array}{c}
s_{1}, s_{2} \\
s_{3} ; \eta
\end{array}\right]=\sum_{m=0}^{\infty} \frac{\left(s_{1}\right)_{m, k}\left(s_{2}\right)_{m, k}}{\left(s_{3}\right)_{m, k}} \cdot \frac{\eta^{m}}{m !},|\eta|<\frac{1}{k}
$$

where $\left(s_{1}\right)_{m, k}$ is the $k$-Pochhammer symbol defined in (4). Obviously, if $k=1$, Equation (6) is reduced to (1).

\section{Hadamard Product of k-Gauss Hypergeometric Functions}

Starting, we define the Hadamard product of k-Gauss hypergeometric functions $\mathcal{H}(k ; \eta)$ as follows:

Definition 4. Suppose that:

$$
{ }_{2} \digamma_{1}^{(k, i)}\left[\begin{array}{c}
\alpha_{i}, \beta_{i} \\
\delta_{i}
\end{array}\right]=\sum_{n=0}^{\infty} \frac{\left(\alpha_{i}\right)_{n, k}\left(\beta_{i}\right)_{n, k}}{\left(\delta_{i}\right)_{n, k} n !} \eta^{n} ; \quad i=1,2, k \in \mathbb{R}^{+},
$$

are two $k$-Gauss hypergeometric functions of the single complex variable $\eta$, as given in (6). Now, we construct the Hadamard product (or convolution) of these two k-Gauss hypergeometric functions, $\left({ }_{2} \digamma_{1}^{(k, 1)} *{ }_{2} \digamma_{1}^{(k, 2)}\right)$ in the form:

$$
\begin{aligned}
\mathcal{H}(k ; \eta) & =\mathcal{H}\left[\begin{array}{c}
\left(\alpha_{1}, k\right),\left(\alpha_{2}, k\right),\left(\beta_{1}, k\right),\left(\beta_{2}, k\right) \\
\left(\delta_{1}, k\right),\left(\delta_{2}, k\right)
\end{array}\right] \\
& =\left({ }_{2} \digamma_{1}^{(k, 1)} *{ }_{2} \digamma_{1}^{(k, 2)}\right)(\eta) \\
& =\sum_{n=0}^{\infty} \frac{\left(\alpha_{1}\right)_{n, k}\left(\beta_{1}\right)_{n, k}\left(\alpha_{2}\right)_{n, k}\left(\beta_{2}\right)_{n, k}}{\left(\delta_{1}\right)_{n, k}\left(\delta_{2}\right)_{n, k} n ! n !} \eta^{n} \\
& =\sum_{n=0}^{\infty} \frac{\left(\alpha_{1}\right)_{n, k}\left(\beta_{1}\right)_{n, k}\left(\alpha_{2}\right)_{n, k}\left(\beta_{2}\right)_{n, k}}{\left(\delta_{1}\right)_{n, k}\left(\delta_{2}\right)_{n, k}(1)_{n} n !} \eta^{n}
\end{aligned}
$$

where $k \in \mathbb{R}^{+}, \alpha_{1}, \alpha_{2}, \beta_{1}, \beta_{2}, \eta \in \mathbb{C}$ and $\delta_{1}, \delta_{2} \in \mathbb{C} \backslash \mathbb{Z}_{0}^{-}$.

Remark 1. Clearly, the Hadamard product of $k$-Gauss hypergeometric functions $\mathcal{H}(k ; \eta)$ is a generalized type of $k$-Gauss hypergeometric function, where:

$$
\mathcal{H}(k ; \eta)={ }_{4} \digamma_{3}\left[\begin{array}{c}
\left(\alpha_{1}, k\right),\left(\alpha_{2}, k\right),\left(\beta_{1}, k\right),\left(\beta_{2}, k\right) \\
\left(\delta_{1}, k\right),\left(\delta_{2}, k\right),(1,1)
\end{array}\right] .
$$


Remark 2. For $k=1$; it obviously reduces to the usual Hadamard product of Gauss hypergeometric functions $\mathcal{H}(1 ; \eta)$, which is a generalized Gauss function in (1).

\subsection{Convergence Property}

Following, we would like to show the convergence property of the series (7). For this purpose, we can rewrite (7) in the form:

$$
\mathcal{H}(k ; \eta)=\mathcal{H}\left[\begin{array}{c}
\left(\alpha_{1}, k\right),\left(\alpha_{2}, k\right),\left(\beta_{1}, k\right),\left(\beta_{2}, k\right) \\
\left(\delta_{1}, k\right),\left(\delta_{2}, k\right)
\end{array}\right]=\sum_{n=0}^{\infty} \mathbf{U}_{n, k}(\eta)
$$

where:

$$
\mathbf{U}_{n, k}(\eta)=\frac{\left(\alpha_{1}\right)_{n, k}\left(\beta_{1}\right)_{n, k}\left(\alpha_{2}\right)_{n, k}\left(\beta_{2}\right)_{n, k}}{\left(\delta_{1}\right)_{n, k}\left(\delta_{2}\right)_{n, k} n ! n !} \eta^{n} .
$$

According to the identity $(x)_{n+1, k}=(x+n k)(x)_{n, k}$, we have:

$$
\frac{\mathbf{U}_{n+1, k}}{\mathbf{U}_{n, k}}=\frac{\left(\alpha_{1}+n k\right)\left(\beta_{1}+n k\right)\left(\alpha_{2}+n k\right)\left(\beta_{2}+n k\right)}{\left(\delta_{1}+n k\right)\left(\delta_{2}+n k\right)(n+1)^{2}} \eta
$$

and hence:

$$
\lim _{n \rightarrow \infty}\left|\frac{\mathbf{U}_{n+1, k}}{\mathbf{U}_{n, k}}\right|=k^{2}|\eta|, \quad \eta \in \mathbb{C}, k \in \mathbb{R}^{+},
$$

therefore, by the ratio test the series on the right-hand side of (7) converges absolutely if $|\eta|<\frac{1}{k^{2}}$ and diverges if $|\eta|>\frac{1}{k^{2}}$.

In the case that $|\eta|=1$, assume that there exists a positive number $\tau$, as:

$$
\tau=\frac{k}{2} \operatorname{Re}\left(\frac{\delta_{1}}{k}+\frac{\delta_{2}}{k}+1-\frac{\alpha_{1}}{k}-\frac{\alpha_{2}}{k}-\frac{\beta_{1}}{k}-\frac{\beta_{2}}{k}\right)>0
$$

and compare terms of the series:

$$
1+\sum_{n=0}^{\infty}\left|\frac{\left(\alpha_{1}\right)_{n, k}\left(\beta_{1}\right)_{n, k}\left(\alpha_{2}\right)_{n, k}\left(\beta_{2}\right)_{n, k}}{\left(\delta_{1}\right)_{n, k}\left(\delta_{2}\right)_{n, k}(1)_{n} n !} \eta^{n}\right|
$$

with corresponding terms of the series:

$$
\sum_{n=0}^{\infty} \frac{1}{(k n)^{1+\frac{\tau}{k}}}, k \in \mathbb{R}^{+}
$$


which is convergent; for $|\eta|=1$, we see that:

$$
\begin{aligned}
& \lim _{n \rightarrow \infty}\left|\frac{(k n)^{1+\frac{\tau}{k}}\left(\alpha_{1}\right)_{n, k}\left(\beta_{1}\right)_{n, k}\left(\alpha_{2}\right)_{n, k}\left(\beta_{2}\right)_{n, k}}{\left(\delta_{1}\right)_{n, k}\left(\delta_{2}\right)_{n, k}(1)_{n} n !} \eta^{n}\right| \\
& =\lim _{n \rightarrow \infty}\left|\frac{\left(\alpha_{1}\right)_{n, k}(n-1) ! k^{n-1}(n k)^{\frac{\alpha_{1}}{k}}(n k)^{\frac{-\alpha_{1}}{k}}}{(n-1) ! k^{n-1}}\right| \lim _{n \rightarrow \infty}\left|\frac{\left(\alpha_{2}\right)_{n, k}(n-1) ! k^{n-1}(n k)^{\frac{\alpha_{2}}{k}}(n k)^{\frac{-\alpha_{2}}{k}}}{(n-1) ! k^{n-1}}\right| \\
& \times \lim _{n \rightarrow \infty}\left|\frac{\left(\beta_{1}\right)_{n, k}(n-1) ! k^{n-1}(n k)^{\frac{\beta_{1}}{k}}(n k)^{\frac{-\beta_{1}}{k}}}{(n-1) ! k^{n-1}}\right| \lim _{n \rightarrow \infty}\left|\frac{\left(\beta_{2}\right)_{n, k}(n-1) ! k^{n-1}(n k)^{\frac{\beta_{2}}{k}}(n k)^{\frac{-\beta_{2}}{k}}}{(n-1) ! k^{n-1}}\right| \\
& \times \lim _{n \rightarrow \infty}\left|\frac{(n k)^{\frac{\delta_{1}}{k}}\left(n k \frac{-\delta_{1}}{k}\right.}{\left(\delta_{1}\right)_{n, k}}\right| \lim _{n \rightarrow \infty}\left|\frac{(n k)^{\frac{\delta_{2}}{k}}(n k)^{\frac{-\delta_{2}}{k}}}{\left(\delta_{2}\right)_{n, k}}\right| \lim _{n \rightarrow \infty}\left|\frac{(k n)^{1+\frac{\tau}{k}}}{(1)_{n} n !}\right| \\
& =\lim _{n \rightarrow \infty}\left|\frac{\Gamma^{k}\left(\delta_{1}\right) \Gamma^{k}\left(\delta_{2}\right)}{\Gamma^{k}\left(\alpha_{1}\right) \Gamma^{k}\left(\alpha_{2}\right) \Gamma^{k}\left(\beta_{1}\right) \Gamma^{k}\left(\beta_{2}\right)}\right| \lim _{n \rightarrow \infty}\left|\frac{k^{2 n-1}}{(n k)^{\frac{\delta_{1}}{k}+\frac{\delta_{2}}{k}}+1-\frac{\alpha_{1}}{k}-\frac{\alpha_{2}}{k}-\frac{\beta_{1}}{k}-\frac{\beta_{2}}{k}-\frac{\tau}{k}}\right|=0,
\end{aligned}
$$

where:

$$
\operatorname{Re}\left(\frac{\delta_{1}}{k}+\frac{\delta_{2}}{k}+1-\frac{\alpha_{1}}{k}-\frac{\alpha_{2}}{k}-\frac{\beta_{1}}{k}-\frac{\beta_{2}}{k}-\frac{\tau}{k}\right)=2 \tau-\tau>0,
$$

thus, the series (7) converges absolutely if $|\eta|=1$, under hypothesis (9).

Summarizing, the following result has been established.

Theorem 1. For $k \in \mathbb{R}^{+}$, the Hadamard product of $k$-Gauss hypergeometric functions $\mathcal{H}(k ; \eta)$ defined in (7),

- Converges absolutely for $|\eta|<\frac{1}{k^{2}}$;

- Converges absolutely for $|\eta|=1$ under condition (9); and

- Diverges for $|\eta|>\frac{1}{k^{2}}$.

Remark 3. For $k=1$ in Theorem 1, we get the convergence property of the generalized Gauss hypergeometric series (see [30,32]).

\subsection{Derivative Formulae}

Theorem 2. The following derivative formula holds true:

$$
\begin{aligned}
& \frac{d^{n}}{d \eta^{n}}\left\{\eta^{\frac{\delta_{1}}{k}} \mathcal{H}\left[\begin{array}{cc}
\left(\alpha_{1}, k\right),\left(\alpha_{2}, k\right),\left(\beta_{1}, k\right),\left(\beta_{2}, k\right) & \\
\left(\delta_{1}+k, k\right),\left(\delta_{2}, k\right) & ; \eta
\end{array}\right]\right\} \\
= & \frac{\eta^{\frac{\delta_{1}}{k}-n} \Gamma^{k}\left(\delta_{1}\right)}{k^{n-1} \Gamma^{k}\left(\delta_{1}-(n-1) k\right)} \mathcal{H}\left[\begin{array}{cc}
\left(\alpha_{1}, k\right),\left(\alpha_{2}, k\right),\left(\beta_{1}, k\right),\left(\beta_{2}, k\right) & ; \eta \\
\left(\delta_{1}-(n-1) k, k\right),\left(\delta_{2}, k\right) & ;
\end{array}\right],
\end{aligned}
$$

$\left(k \in \mathbb{R}^{+}, \operatorname{Re}\left(\alpha_{1}\right)>0, \operatorname{Re}\left(\alpha_{2}\right)>0, \operatorname{Re}\left(\beta_{1}\right)>0, \operatorname{Re}\left(\beta_{2}\right)>0, \operatorname{Re}\left(\delta_{1}\right)>0, \operatorname{Re}\left(\delta_{2}\right)>0,|\eta|<\right.$ $\left.\frac{1}{k^{2}}, n \in \mathbb{N}_{0}\right)$.

Proof. Result (13) is obviously valid in the trivial case when $n=0$. For $n=1$, by the power series representation $(7)$ of $\mathcal{H}(k ; \eta)$, we see from (13) that:

$$
\begin{aligned}
& \frac{d}{d \eta}\left\{\eta^{\frac{\delta_{1}}{k}} \sum_{n=0}^{\infty} \frac{\left(\alpha_{1}\right)_{n, k}\left(\beta_{1}\right)_{n, k}\left(\alpha_{2}\right)_{n, k}\left(\beta_{2}\right)_{n, k}}{\left(\delta_{1}+k\right)_{n, k}\left(\delta_{2}\right)_{n, k} n ! n !} \eta^{n}\right\} \\
= & \sum_{n=0}^{\infty} \frac{\left(\alpha_{1}\right)_{n, k}\left(\beta_{1}\right)_{n, k}\left(\alpha_{2}\right)_{n, k}\left(\beta_{2}\right)_{n, k}\left(\delta_{1}+n k\right)}{\left(\delta_{1}+k\right)_{n, k}\left(\delta_{2}\right)_{n, k} k n ! n !} \eta^{\frac{\delta_{1}}{k}+n-1} .
\end{aligned}
$$


Replacing the k-pochhammer symbole $\left(\delta_{1}+k\right)_{n, k}$ in the relation (4) by its k-gamma function and using the k-gamma function property given in [18] by $\Gamma^{k}\left(\delta_{1}+k\right)=\delta_{1} \Gamma^{k}\left(\delta_{1}\right)$, we arrive at:

$$
\begin{aligned}
& \frac{d}{d \eta}\left\{\eta^{\frac{\delta_{1}}{k}} \sum_{n=0}^{\infty} \frac{\left(\alpha_{1}\right)_{n, k}\left(\beta_{1}\right)_{n, k}\left(\alpha_{2}\right)_{n, k}\left(\beta_{2}\right)_{n, k}}{\left(\delta_{1}+k\right)_{n, k}\left(\delta_{2}\right)_{n, k} n ! n !} \eta^{n}\right\} \\
= & \frac{\delta_{1}}{k} \eta^{\frac{\delta_{1}}{k}-1} \mathcal{H}\left[\begin{array}{c}
\left(\alpha_{1}, k\right),\left(\alpha_{2}, k\right),\left(\beta_{1}, k\right),\left(\beta_{2}, k\right) \\
\left(\delta_{1}, k\right),\left(\delta_{2}, k\right)
\end{array} ; .\right.
\end{aligned}
$$

Therefore, the general result (13) can now be easily derived by using the principle of mathematical induction on $n \in \mathbb{N}_{0}$.

Similarly, we can derive the following results.

Theorem 3. The following derivative formula holds true:

$$
\begin{aligned}
& \frac{d^{n}}{d \eta^{n}}\left\{\eta^{\frac{\delta_{2}}{k}} \mathcal{H}\left[\begin{array}{r}
\left(\alpha_{1}, k\right),\left(\alpha_{2}, k\right),\left(\beta_{1}, k\right),\left(\beta_{2}, k\right) \\
\left(\delta_{1}, k\right),\left(\delta_{2}+k, k\right)
\end{array}\right]\right\} \\
&= \frac{\eta^{\frac{\delta_{2}}{k}-n} \Gamma^{k}\left(\delta_{2}\right)}{k^{n-1} \Gamma^{k}\left(\delta_{2}-(n-1) k\right)} \mathcal{H}\left[\begin{array}{c}
\left(\alpha_{1}, k\right),\left(\alpha_{2}, k\right),\left(\beta_{1}, k\right),\left(\beta_{2}, k\right) \\
\left(\delta_{1}, k\right),\left(\delta_{2}-(n-1) k, k\right)
\end{array}\right], \\
&\left(k \in \mathbb{R}^{+}, \operatorname{Re}\left(\alpha_{1}\right)>0, \operatorname{Re}\left(\alpha_{2}\right)>0, \operatorname{Re}\left(\beta_{1}\right)>0, \operatorname{Re}\left(\beta_{2}\right)>0, \operatorname{Re}\left(\delta_{1}\right)>0, \operatorname{Re}\left(\delta_{2}\right)>\right.
\end{aligned}
$$
$\left.0,|\eta|<\frac{1}{k^{2}}, n \in \mathbb{N}_{0}\right)$.

Remark 4. At $k=1$ in (13) and (16), we obtain differentiation formulae of the generalized hypergeometric function (cf. [32]).

\section{Some Integral Representations and Integral Transforms}

In this section, we evaluate the integral representations and integral transforms for the Hadamard product of k-Gauss hypergeometric functions $\mathcal{H}(k ; \eta)$.

\subsection{Integral Representations}

Theorem 4. The Hadamard product of $k$-Gauss hypergeometric functions $\mathcal{H}(k ; \eta)$ has the following integral representations:

$$
\begin{aligned}
& \mathcal{H}(k ; \eta)=\mathcal{H}\left[\begin{array}{c}
\left(\alpha_{1}, k\right),\left(\alpha_{2}, k\right),\left(\beta_{1}, k\right),\left(\beta_{2}, k\right) \\
\left(\delta_{1}, k\right),\left(\delta_{2}, k\right)
\end{array}\right] \\
& =\frac{1}{\Gamma k\left(\alpha_{1}\right)} \int_{0}^{\infty} u^{\alpha_{1}-1} e^{-\frac{u^{k}}{k}}{ }_{3} \digamma_{3}\left[\begin{array}{r}
\left(\alpha_{2}, k\right),\left(\beta_{1}, k\right),\left(\beta_{2}, k\right) \\
\left(\delta_{1}, k\right),\left(\delta_{2}, k\right),(1,1)
\end{array}\right] d u \\
& \left(|\eta|<\frac{1}{k^{2}}, \operatorname{Re}\left(\alpha_{1}\right)>0, k \in \mathbb{R}^{+}\right) . \\
& \mathcal{H}(k ; \eta)=\mathcal{H}\left[\begin{array}{c}
\left(\alpha_{1}, k\right),\left(\alpha_{2}, k\right),\left(\beta_{1}, k\right),\left(\beta_{2}, k\right) \\
\left(\delta_{1}, k\right),\left(\delta_{2}, k\right)
\end{array}\right] \\
& =\frac{1}{k \mathbf{B}^{k}\left(\beta_{1}, \delta_{1}-\beta_{1}\right)} \int_{0}^{1} u^{\frac{\beta_{1}}{k}-1}(1-u)^{\frac{\delta_{1}-\beta_{1}}{k}-1}{ }_{3} \digamma_{2}\left[\begin{array}{c}
\left(\alpha_{1}, k\right),\left(\alpha_{2}, k\right),\left(\beta_{2}, k\right) \\
\left(\delta_{2}, k\right),(1,1)
\end{array}\right] d u
\end{aligned}
$$




$$
\begin{aligned}
& \left(|\eta|<\frac{1}{k^{2}}, \operatorname{Re}\left(\delta_{1}\right)>\beta_{1}>0, k \in \mathbb{R}^{+}\right) . \\
& \mathcal{H}(k ; \eta)=\mathcal{H}\left[\begin{array}{c}
\left(\alpha_{1}, k\right),\left(\alpha_{2}, k\right),\left(\beta_{1}, k\right),\left(\beta_{2}, k\right) \\
\left(\delta_{1}, k\right),\left(\delta_{2}, k\right)
\end{array}\right] \\
& =\frac{1}{k^{2} \mathbf{B}^{k}\left(\alpha_{2}, \delta_{1}-\alpha_{2}\right) \mathbf{B}^{k}\left(\beta_{2}, \delta_{2}-\beta_{2}\right)} \\
& \times \int_{0}^{1} \int_{0}^{1} v^{\frac{\alpha_{2}}{k}-1}(1-v)^{\frac{\delta_{1}-\alpha_{2}}{k}-1} u^{\frac{\beta_{2}}{k}-1}(1-u)^{\frac{\delta_{2}-\beta_{2}}{k}-1}{ }_{2} \digamma_{1}\left[\begin{array}{c}
\left(\alpha_{1}, k\right),\left(\beta_{1}, k\right) \\
(1,1)
\end{array}\right] d v d u \\
& \quad\left(|\eta|<\frac{1}{k^{2}}, \operatorname{Re}\left(\delta_{1}\right)>\operatorname{Re}\left(\alpha_{2}\right)>0, \operatorname{Re}\left(\delta_{2}\right)>\operatorname{Re}\left(\beta_{2}\right)>0, k \in \mathbb{R}^{+}\right) .
\end{aligned}
$$

Proof. Inserting the k-Pochhammer symbol $\left(\alpha_{1}\right)_{n, k}$ from (4) into definition (4) by its integral form given by (3) and from the relation (8), we thus obtain the desired result (17).

The integral form (18) involves the k-beta function $\mathbf{B}^{k}(u, v)$ defined in (5). Thus, the integral formula (18) can indeed be easily obtained by (8) and using the following identity:

$$
\begin{aligned}
\frac{\left(\beta_{1}\right)_{n, k}}{\left(\delta_{1}\right)_{n, k}} & =\frac{\mathbf{B}^{k}\left(\beta_{1}+k n, \delta_{1}-\beta_{1}\right)}{\mathbf{B}^{k}\left(\beta_{1}, \delta_{1}-\beta_{1}\right)} \\
& =\frac{1}{k \mathbf{B}^{k}\left(\beta_{1}, \delta_{1}-\beta_{1}\right)} \int_{0}^{\infty} u^{\frac{\beta_{1}+k n}{k}-1}(1-u)^{\frac{\delta_{1}-\beta_{1}}{k}-1} d u .
\end{aligned}
$$

Now, we prove integral Formula (19). According to relation (20), we can write that:

$$
\frac{\left(\alpha_{2}\right)_{n, k}}{\left(\delta_{1}\right)_{n, k}}=\frac{1}{k \mathbf{B}^{k}\left(\alpha_{2}, \delta_{1}-\alpha_{2}\right)} \int_{0}^{\infty} u^{\frac{\alpha_{2}+k n}{k}-1}(1-u)^{\frac{\delta_{1}-\alpha_{2}}{k}-1} d u
$$

and:

$$
\frac{\left(\beta_{2}\right)_{n, k}}{\left(\delta_{2}\right)_{n, k}}=\frac{1}{k \mathbf{B}^{k}\left(\beta_{2}, \delta_{2}-\beta_{2}\right)} \int_{0}^{\infty} v^{\frac{\beta_{2}+k n}{k}-1}(1-v)^{\frac{\delta_{2}-\beta_{2}}{k}-1} d v
$$

We insert Formulas (21) and (22) into the definition of $\mathcal{H}(k ; \eta)$ in (7) and by (8), and it follows:

$$
\begin{aligned}
\mathcal{H}(k ; \eta)= & \mathcal{H}\left[\begin{array}{c}
\left(\alpha_{1}, k\right),\left(\alpha_{2}, k\right),\left(\beta_{1}, k\right),\left(\beta_{2}, k\right) \\
\left(\delta_{1}, k\right),\left(\delta_{2}, k\right)
\end{array}\right] \\
= & \frac{1}{k^{2} \mathbf{B}^{k}\left(\alpha_{2}, \delta_{1}-\alpha_{2}\right) \mathbf{B}^{k}\left(\beta_{2}, \delta_{2}-\beta_{2}\right)} \sum_{n=0}^{\infty}\left(\alpha_{1}\right)_{n, k}\left(\beta_{1}\right)_{n, k} \\
& \times\left(\int_{0}^{\infty} u^{\frac{\alpha_{2}+k n}{k}-1}(1-u)^{\frac{\delta_{1}-\alpha_{2}}{k}-1} d u\right) \\
& \times\left(\int_{0}^{\infty} v^{\frac{\beta_{2}+k n}{k}-1}(1-v)^{\frac{\delta_{2}-\beta_{2}}{k}-1} d v\right) \frac{\eta^{n}}{n ! n !} \\
& =\frac{1}{k^{2} \mathbf{B}^{k}\left(\alpha_{2}, \delta_{1}-\alpha_{2}\right) \mathbf{B}^{k}\left(\beta_{2}, \delta_{2}-\beta_{2}\right)} \\
& \times \int_{0}^{1} \int_{0}^{1} v^{\frac{\beta_{2}}{k}-1} u^{\frac{\alpha_{2}}{k}-1}(1-v)^{\frac{\delta_{2}-\beta_{2}}{k}-1}(1-u)^{\frac{\delta_{1}-\alpha_{2}}{k}-1} \\
& \times{ }_{2} \digamma_{1}\left[\begin{array}{c}
\left(\alpha_{1}, k\right),\left(\beta_{1}, k\right) \\
(1,1)
\end{array}\right] d v d u
\end{aligned}
$$


which completes the proof (19).

Remark 5. The obtained results here improve and generalize some known results in [18,21].

Remark 6. For $k=1$ in Theorem 4, we get interesting results concerning integral formulae of the generalized hypergeometric function (see [32]).

\subsection{Integral Transforms}

In this subsection, we introduce certain interesting k-Beta transforms, Laplace transforms, and inverse Laplace transforms associated with the Hadamard product of k-Gauss hypergeometric functions $\mathcal{H}(k ; \eta)$. We recall the $\mathrm{k}$-Beta transform, the Laplace transform and inverse Laplace transform of $G(\eta)$, respectively:

$$
\begin{gathered}
\mathbb{B}_{k}\left\{G(\eta): \alpha_{1}, \beta_{1}\right\}=\frac{1}{k} \int_{0}^{1} u^{\frac{\alpha_{1}}{k}-1}(1-u)^{\frac{\beta_{1}}{k}-1} G(\eta) d u, \\
\left(\operatorname{Re}\left(\alpha_{1}\right)>0, \operatorname{Re}\left(\beta_{1}\right)>0,|\eta|<\frac{1}{k^{2}}, k \in \mathbb{R}^{+}\right), \\
\mathbb{L}\{G(\eta): u\}=G(u)=\int_{0}^{\infty} e^{-u \eta} G(\eta) d \eta, \quad \operatorname{Re}(u)>0,
\end{gathered}
$$

provided that the integral converges. For simplicity, we can write it thus:

$$
G(\eta)=\mathbb{L}^{-1}\{G(u)\} .
$$

Theorem 5. The k-Beta transform for the Hadamard product of $k$-Gauss hypergeometric functions $\mathcal{H}(k ; \eta)$ in $(7)$ is given in the following form:

$$
\begin{gathered}
\mathbb{B}_{k}\left(\mathcal{H}\left[\begin{array}{c}
\left(\alpha_{1}+\beta_{1}, k\right),\left(\alpha_{2}, k\right),\left(\beta_{1}, k\right),\left(\beta_{2}, k\right) \\
\left(\delta_{1}, k\right),\left(\delta_{2}, k\right)
\end{array}\right]: \alpha_{1}, \beta_{1}\right) \\
=\mathbf{B}^{k}\left(\alpha_{1}, \beta_{1}\right)\left[\mathcal{H}\left[\begin{array}{c}
\left(\alpha_{1}, k\right),\left(\alpha_{2}, k\right),\left(\beta_{1}, k\right),\left(\beta_{2}, k\right) \\
\left(\delta_{1}, k\right),\left(\delta_{2}, k\right)
\end{array}\right]\right], \\
\left(k \in \mathbb{R}^{+}, \operatorname{Re}\left(\alpha_{1}\right)>0, \operatorname{Re}\left(\alpha_{2}\right)>0, \operatorname{Re}\left(\beta_{1}\right)>0, \operatorname{Re}\left(\beta_{2}\right)>0, \operatorname{Re}\left(\delta_{1}\right)>0, \operatorname{Re}\left(\delta_{2}>0\right),\right.
\end{gathered}
$$$$
\left.|\eta|<\frac{1}{k^{2}}\right) \text {. }
$$

Proof. Applying the k-Beta transform (24) to (7), we find that:

$$
\begin{aligned}
& \mathbb{B}_{k}\left(\mathcal{H}\left[\begin{array}{c}
\left(\alpha_{1}+\beta_{1}, k\right),\left(\alpha_{2}, k\right),\left(\beta_{1}, k\right),\left(\beta_{2}, k\right) \\
\left(\delta_{1}, k\right),\left(\delta_{2}, k\right)
\end{array}\right]: \alpha_{1}, \beta_{1}\right) \\
= & \frac{1}{k} \int_{0}^{1} u^{\frac{\alpha_{1}}{k}-1}(1-u)^{\frac{\beta_{1}}{k}-1} \mathcal{H}\left[\begin{array}{c}
\left(\alpha_{1}+\beta_{1}, k\right),\left(\alpha_{2}, k\right),\left(\beta_{1}, k\right),\left(\beta_{2}, k\right) \\
\left(\delta_{1}, k\right),\left(\delta_{2}, k\right)
\end{array}\right] d u \\
= & \frac{1}{k} \int_{0}^{1} u^{\frac{\alpha_{1}}{k}-1}(1-u)^{\frac{\beta_{1}}{k}-1} \sum_{n=0}^{\infty} \frac{\left(\alpha_{1}+\beta_{1}\right)_{n, k}\left(\beta_{1}\right)_{n, k}\left(\alpha_{2}\right)_{n, k}\left(\beta_{2}\right)_{n, k}}{\left(\delta_{1}\right)_{n, k}\left(\delta_{2}\right)_{n, k} n ! n !}(u \eta)^{n} d u,
\end{aligned}
$$


which is given in terms of the k-beta function $\mathbf{B}^{k}\left(\alpha_{1}, \beta_{1}\right)$ in the form:

$$
\begin{aligned}
& \mathbb{B}_{k}\left(\mathcal{H}\left[\begin{array}{c}
\left(\alpha_{1}+\beta_{1}, k\right),\left(\alpha_{2}, k\right),\left(\beta_{1}, k\right),\left(\beta_{2}, k\right) \\
\left(\delta_{1}, k\right),\left(\delta_{2}, k\right)
\end{array} ; \eta\right]: \alpha_{1}, \beta_{1}\right) \\
= & \sum_{n=0}^{\infty} \frac{\mathbf{B}^{k}\left(\alpha_{1}+n k, \beta_{1}\right)\left(\alpha_{1}+\beta_{1}\right)_{n, k}\left(\beta_{1}\right)_{n, k}\left(\alpha_{2}\right)_{n, k}\left(\beta_{2}\right)_{n, k}}{\left(\delta_{1}\right)_{n, k}\left(\delta_{2}\right)_{n, k} n ! n !} \eta^{n} d u \\
= & \mathbf{B}^{k}\left(\alpha_{1} \beta_{1}\right) \sum_{n=0}^{\infty} \frac{\left(\alpha_{1}\right)_{n, k}\left(\beta_{1}\right)_{n, k}\left(\alpha_{2}\right)_{n, k}\left(\beta_{2}\right)_{n, k}}{\left(\delta_{1}\right)_{n, k}\left(\delta_{2}\right)_{n, k} n ! n !} \eta^{n} .
\end{aligned}
$$

According to (7), our desired result is yielded (27).

Remark 7. If we set $k=1$ then our result reduces to the result of the Beta transform of the generalized hypergeometric function.

Theorem 6. For $k \in \mathbb{R}^{+}, \operatorname{Re}(u)>0$ and $\left|\frac{\eta}{u}\right|<1$, the following Laplace transform formula holds:

$$
\begin{aligned}
& \mathbb{L}\left\{\mathcal{H}\left[\begin{array}{c}
\left(\alpha_{1}, k\right),\left(\alpha_{2}, k\right),\left(\beta_{1}, k\right),\left(\beta_{2}, k\right) \\
\left(\delta_{1}, k\right),\left(\delta_{2}, k\right)
\end{array} ; v\right]: u\right\} \\
= & \frac{1}{u}\left\{{ }_{5} \digamma_{3}\left[\begin{array}{c}
\left(\alpha_{1}, k\right),(k, k) ;\left(\alpha_{2}, k\right),\left(\beta_{1}, k\right),\left(\beta_{2}, k\right) \\
\left(\delta_{1}, k\right),\left(\delta_{2}, k\right),(1,1)
\end{array} ; \frac{\eta}{k u}\right]\right\} .
\end{aligned}
$$

Proof. Using (7) on the above left-hand side integral transform we observe that:

$$
\begin{aligned}
& \mathbb{L}\left\{\mathcal{H}\left[\begin{array}{c}
\left(\alpha_{1}, k\right),\left(\alpha_{2}, k\right),\left(\beta_{1}, k\right),\left(\beta_{2}, k\right) \\
\left(\delta_{1}, k\right),\left(\delta_{2}, k\right)
\end{array}\right]: u\right\} \\
= & \int_{0}^{\infty} e^{-u v} \sum_{n=0}^{\infty} \frac{\left(\beta_{1}\right)_{n, k}\left(\alpha_{2}\right)_{n, k}\left(\beta_{2}\right)_{n, k}}{\left(\delta_{1}\right)_{n, k}\left(\delta_{2}\right)_{n, k} n ! n !}(v \eta)^{n} d v \\
= & \sum_{n=0}^{\infty} \frac{\left(\beta_{1}\right)_{n, k}\left(\alpha_{2}\right)_{n, k}\left(\beta_{2}\right)_{n, k}}{\left(\delta_{1}\right)_{n, k}\left(\delta_{2}\right)_{n, k} n ! n !}(\eta)^{n} \int_{0}^{\infty} e^{-u v} v^{n} d v .
\end{aligned}
$$

Setting $u v=\frac{t^{k}}{k}$, then applying the k-gamma function (6) to the last integral, upon using (8), yields our desired result (30), as follows:

$$
\begin{aligned}
& \mathbb{L}\left\{\mathcal{H}\left[\begin{array}{c}
\left.\left.\left(\alpha_{1}, k\right),\left(\alpha_{2}, k\right),\left(\beta_{1}, k\right),\left(\beta_{2}, k\right) ; \eta v\right]: u\right\} \\
\left(\delta_{1}, k\right),\left(\delta_{2}, k\right)
\end{array}\right]\right. \\
= & \sum_{n=0}^{\infty} \frac{\left(\beta_{1}\right)_{n, k}\left(\alpha_{2}\right)_{n, k}\left(\beta_{2}\right)_{n, k}}{\left(\delta_{1}\right)_{n, k}\left(\delta_{2}\right)_{n, k} n ! n ! k^{n} u^{n+1}}(\eta)^{n} \Gamma(n k+k) \\
= & \frac{1}{u}\left\{{ }_{5} \digamma_{3}\left[\begin{array}{c}
\left(\alpha_{1}, k\right),(k, k) ;\left(\alpha_{2}, k\right),\left(\beta_{1}, k\right),\left(\beta_{2}, k\right) \\
\left(\delta_{1}, k\right),\left(\delta_{2}, k\right),(1,1)
\end{array} ; \frac{\eta}{k u}\right]\right\} .
\end{aligned}
$$


Theorem 7. If $k \in \mathbb{R}^{+}, \operatorname{Re}\left(\alpha_{1}\right)>0, \operatorname{Re}\left(\alpha_{2}\right)>0, \operatorname{Re}\left(\beta_{1}\right)>0, \operatorname{Re}\left(\beta_{2}\right)>0, \operatorname{Re}\left(\delta_{1}\right)>0$, $\operatorname{Re}\left(\delta_{2}\right)>0$, and $\left|\eta v^{k}\right|<1$, then the following inverse Laplace transform formula holds:

$$
\begin{aligned}
& \mathbb{L}^{-1}\left\{u^{-\alpha_{1}} \mathcal{H}\left[\begin{array}{c}
\left(\alpha_{1}, k\right),\left(\alpha_{2}, k\right),\left(\beta_{1}, k\right),\left(\beta_{2}, k\right) \\
\left(\delta_{1}, k\right),\left(\delta_{2}, k\right)
\end{array} ; \frac{\eta}{u^{k}}\right]\right\} \\
= & \frac{v^{\alpha_{1}-1}}{\Gamma^{k}\left(\alpha_{1}\right)} \mathcal{H}\left[\begin{array}{c}
\left(\alpha_{2}, k\right),\left(\beta_{1}, k\right),\left(\beta_{2}, k\right) \\
\left(\delta_{1}, k\right),\left(\delta_{2}, k\right)
\end{array} ; v^{k}\right] .
\end{aligned}
$$

Proof. Using the definition of the Hadamard product of k-Gauss hypergeometric functions by (7) in the left-hand side of (33), we have:

$$
\begin{aligned}
& \mathbb{L}^{-1}\left\{u^{-\alpha_{1}} \mathcal{H}\left[\begin{array}{c}
\left(\alpha_{1}, k\right),\left(\alpha_{2}, k\right),\left(\beta_{1}, k\right),\left(\beta_{2}, k\right) \\
\left(\delta_{1}, k\right),\left(\delta_{2}, k\right)
\end{array} \frac{\eta}{u^{k}}\right]\right\} \\
= & \sum_{n=0}^{\infty} \frac{\left(\alpha_{1}\right)_{n, k}\left(\beta_{1}\right)_{n, k}\left(\alpha_{2}\right)_{n, k}\left(\beta_{2}\right)_{n, k}}{\left(\delta_{1}\right)_{n, k}\left(\delta_{2}\right)_{n, k} n ! n !} \eta^{n} \mathbb{L}^{-1}\left\{u^{-\left(\alpha_{1}+n k\right)}\right\} .
\end{aligned}
$$

Applying the inverse Laplace transform and after simplification, we find that:

$$
\begin{aligned}
& \mathbb{L}^{-1}\left\{u^{-\alpha_{1}} \mathcal{H}\left[\begin{array}{c}
\left(\alpha_{1}, k\right),\left(\alpha_{2}, k\right),\left(\beta_{1}, k\right),\left(\beta_{2}, k\right) \\
\left(\delta_{1}, k\right),\left(\delta_{2}, k\right)
\end{array} ; \frac{\eta}{u^{k}}\right]\right\} \\
= & \sum_{n=0}^{\infty} \frac{\left(\alpha_{1}\right)_{n, k}\left(\beta_{1}\right)_{n, k}\left(\alpha_{2}\right)_{n, k}\left(\beta_{2}\right)_{n, k}}{\left(\delta_{1}\right)_{n, k}\left(\delta_{2}\right)_{n, k} n ! n !} \eta^{n} \frac{v^{\alpha_{1}+n k-1}}{\Gamma^{k}\left(\alpha_{1}+n k\right)} \\
= & \frac{v^{\alpha_{1}-1}}{\Gamma^{k}\left(\alpha_{1}\right)} \mathcal{H}\left[\begin{array}{c}
\left(\alpha_{2}, k\right),\left(\beta_{1}, k\right),\left(\beta_{2}, k\right) \\
\left(\delta_{1}, k\right),\left(\delta_{2}, k\right)
\end{array} ; \eta v^{k}\right] .
\end{aligned}
$$

The above equation gives the proof of Theorem 7 .

Remark 8. The special cases of (30) and (33) when $k=1$ are seen to reduce to the Laplace transform and inverse Laplace transform of the generalized hypergeometric functions (see $[33,34])$.

\section{Contiguous Function Relations and Differential Equations}

The k-analogue of theta operator $k \Theta$ as given in $[19,20,27]$ takes the form $k \Theta=k \frac{\eta}{d \eta}$. This operator has the particularly pleasant property that $k \Theta \eta^{n}=k n \eta^{n}$, which makes it handy to be used on power series. In this section, relying on Definition 2.1, we present some results concerning contiguous function relations and differential equations for the HPkGHF.

To realize that, we increase or decrease one and more of the parameters of the Hadamard product of k-Gauss hypergeometric functions,

$$
\begin{aligned}
\mathcal{H}(k ; \eta) & =\mathcal{H}\left[\begin{array}{c}
\left(\alpha_{1}, k\right),\left(\alpha_{2}, k\right),\left(\beta_{1}, k\right),\left(\beta_{2}, k\right) \\
\left(\delta_{1}, k\right),\left(\delta_{2}, k\right)
\end{array}\right] \\
& =\sum_{n=0}^{\infty} \frac{\left(\alpha_{1}\right)_{n, k}\left(\beta_{1}\right)_{n, k}\left(\alpha_{2}\right)_{n, k}\left(\beta_{2}\right)_{n, k}}{\left(\delta_{1}\right)_{n, k}\left(\delta_{2}\right)_{n, k} n ! n !} \eta^{n}, k \in \mathbb{R}^{+},
\end{aligned}
$$


by $\pm k$, then the resultant function is said to be contiguous to $\mathcal{H}(k ; \eta)$. For simplicity, we use the following notations:

$$
\mathcal{H}\left(k ; \alpha_{1} \pm\right)=\mathcal{H}\left[\begin{array}{cc}
\left(\alpha_{1} \pm k, k\right),\left(\alpha_{2}, k\right),\left(\beta_{1}, k\right),\left(\beta_{2}, k\right) & \\
\left(\delta_{1}, k\right),\left(\delta_{2}, k\right) & ; \eta
\end{array}\right] .
$$

Now, we consider:

$$
\begin{aligned}
\mathcal{H}\left(k ; \alpha_{1}+\right) & =\sum_{n=0}^{\infty} \frac{\left(\alpha_{1}+k\right)_{n, k}\left(\beta_{1}\right)_{n, k}\left(\alpha_{2}\right)_{n, k}\left(\beta_{2}\right)_{n, k}}{\left(\delta_{1}\right)_{n, k}\left(\delta_{2}\right)_{n, k} n ! n !} \eta^{n} \\
= & \sum_{n=0}^{\infty} \frac{\left(\alpha_{1}+n k\right)}{\alpha_{1}} \mathbf{U}_{n, k}, \quad k \in \mathbb{R}^{+}
\end{aligned}
$$

where $\alpha_{1}\left(\alpha_{1}+k\right)_{n, k}=\left(\alpha_{1}+n k\right)\left(\alpha_{1}\right)_{n, k}$ and:

$$
\mathbf{U}_{n, k}(\eta)=\frac{\left(\alpha_{1}\right)_{n, k}\left(\beta_{1}\right)_{n, k}\left(\alpha_{2}\right)_{n, k}\left(\beta_{2}\right)_{n, k}}{\left(\delta_{1}\right)_{n, k}\left(\delta_{2}\right)_{n, k} n ! n !} \eta^{n} .
$$

Similarly, we can write $\mathcal{H}\left(k ; \alpha_{1}-\right)$ as:

$$
\mathcal{H}\left(k ; \alpha_{1}-\right)=\sum_{n=0}^{\infty} \frac{(\alpha-k)}{\left(\alpha_{1}(n-1) k\right)} \mathbf{U}_{n, k}
$$

where $\left(\alpha_{1}(n-1) k\right)\left(\alpha_{1}-k\right)_{n, k}=(\alpha-k)\left(\alpha_{1}\right)_{n, k}$.

Similarly, this holds for $\mathcal{H}\left(k ; \alpha_{2} \pm\right), \mathcal{H}\left(k ; \beta_{1} \pm\right), \mathcal{H}\left(k ; \beta_{2} \pm\right), \mathcal{H}\left(k ; \delta_{1} \pm\right)$, and $\mathcal{H}\left(k ; \delta_{2} \pm\right)$.

By the help of differential operator $k \Theta=k \frac{\eta}{d \eta}$, we get the following relations:

$$
\begin{aligned}
\left(k \Theta+\alpha_{1}\right) \mathcal{H} & =\alpha_{1} \mathcal{H}\left(k ; \alpha_{1}+\right) \\
\left(k \Theta+\beta_{1}\right) \mathcal{H} & =\beta_{1} H\left(\beta_{1}+k\right) \\
\left(k \Theta+\alpha_{2}\right) \mathcal{H} & =\alpha_{2} \mathcal{H}\left(k ; \alpha_{2}+\right) \\
\left(k \Theta+\beta_{2}\right) \mathcal{H} & =\beta_{2} \mathcal{H}\left(k ; \beta_{2}+\right) \\
\left(k \Theta+\delta_{1}-k\right) \mathcal{H} & =\left(\delta_{1}-k\right) \mathcal{H}\left(k ; \delta_{1}-\right) \\
\left(k \Theta+\delta_{2}-k\right) \mathcal{H} & =\left(\delta_{2}-k\right) \mathcal{H}\left(k ; \delta_{2}-\right)
\end{aligned}
$$

From the above relations, we can easily obtain the following results:

$$
\begin{gathered}
\left(\alpha_{1}-\beta_{1}\right) \mathcal{H}(k ; \eta)=\alpha_{1} \mathcal{H}\left(k ; \alpha_{1}+\right)-\beta_{1} \mathcal{H}\left(k ; \beta_{1}+\right), \\
\left(\alpha_{1}-\alpha_{2}\right) \mathcal{H}(k ; \eta)=\alpha_{1} \mathcal{H}\left(k ; \alpha_{1}+\right)-\alpha_{2} \mathcal{H}\left(k ; \alpha_{2}+2 k\right), \\
\left(\beta_{1}-\beta_{2}\right) \mathcal{H}(k ; \eta)=\beta_{1} \mathcal{H}\left(k ; \beta_{1}+, \beta_{2}+\right)-\beta_{2} \mathcal{H}\left(k ; \beta_{2}+\right), \\
\left(\alpha_{1}-\delta_{1}+k\right) \mathcal{H}(k ; \eta)=\alpha_{1} \mathcal{H}\left(k ; \alpha_{1}+\right)-\left(\delta_{1}-k\right) \mathcal{H}\left(k ; \alpha_{2}+2 k\right), \\
\left(\alpha_{2}-\delta_{2}+k\right) \mathcal{H}(k ; \eta)=\alpha_{2} \mathcal{H}\left(k ; \alpha_{2}+\right)-\left(\delta_{2}-k\right) \mathcal{H}\left(k ; \delta_{2}-\right),
\end{gathered}
$$

where:

$$
\mathcal{H}\left(k ; \beta_{1}+, \beta_{2}+\right)=\sum_{n=0}^{\infty} \frac{\left(\alpha_{1}\right)_{n, k}\left(\beta_{1}+k\right)_{n, k}\left(\alpha_{2}\right)_{n, k}\left(\beta_{2}+k\right)_{n, k}}{\left(\delta_{1}\right)_{n, k}\left(\delta_{2}\right)_{n, k} n ! n !} \eta^{n},
$$


is contiguous function relation for the Hadamard product of the k-Gauss hypergeometric function's two parameters, and:

$$
\mathcal{H}\left(k ; \alpha_{1}+2 k\right)=\mathcal{H}\left[\begin{array}{cc}
\left(\alpha_{1}+2 k, k\right),\left(\alpha_{2}, k\right),\left(\beta_{1}, k\right),\left(\beta_{2}, k\right) & ; \eta . \\
\left(\delta_{1}, k\right),\left(\delta_{2}, k\right) &
\end{array}\right] .
$$

Remark 9. It is easy to see that in (36) to (40), if we take $k=1$, we get generalized hypergeometric contiguous function relations (see [32]).

Remark 10. Other contiguous function relations for the Hadamard product of the $k$-Gauss hypergeometric function may be derived from the relations in (36) to (40), and in the same manner, other results can also be obtained.

Remark 11. We can easily obtain many known results in [20,24] by setting the parameters in our main findings. Therefore, the obtained results here extend to those results.

Furthermore, the operator $k \Theta=k \frac{\eta}{d \eta}$, which is used in the derivation of the contiguous function relations, is also used in deriving the differential equations satisfied by $\mathcal{H}(k ; \eta)$ and its contiguous function relations as follows:

$$
\begin{aligned}
k^{2} \Theta^{2} \mathcal{H}(k, \eta) & =\sum_{n=1}^{\infty} \frac{n^{2} k^{2}\left(\alpha_{1}\right)_{n, k}\left(\alpha_{2}\right)_{n, k}\left(\beta_{1}\right)_{n, k}\left(\beta_{2}\right)_{n, k}}{\left(\delta_{1}\right)_{n, k}\left(\delta_{2}\right)_{n, k} n ! n !} \eta^{n} \\
& =\sum_{n=1}^{\infty} \frac{k^{2}\left(\alpha_{1}\right)_{n, k}\left(\alpha_{2}\right)_{n, k}\left(\beta_{1}\right)_{n, k}\left(\beta_{2}\right)_{n, k}}{\left(\delta_{1}\right)_{n, k}\left(\delta_{2}\right)_{n, k}(n-1) !(n-1) !} \eta^{n} \\
& =\sum_{n=0}^{\infty} \frac{k^{2}\left(\alpha_{1}\right)_{n+1, k}\left(\alpha_{2}\right)_{n+1, k}\left(\beta_{1}\right)_{n+1, k}\left(\beta_{2}\right)_{n+1, k}}{\left(\delta_{1}\right)_{n+1, k}\left(\delta_{2}\right)_{n+1, k} n ! n !} \eta^{n}
\end{aligned}
$$

using the following identity $(a)_{n+1, k}=(a)_{n, k}(a+n k)$, we find that:

$$
k^{2} \Theta^{2} \mathcal{H}(k, \eta)=k^{2} \eta \sum_{n=0}^{\infty} \frac{\left(\alpha_{1}+n k\right)\left(\alpha_{2}+n k\right)\left(\beta_{1}+n k\right)\left(\beta_{2}+n k\right)}{\left(\delta_{1}+n k\right)\left(\delta_{2}+n k\right)} \mathbf{U}_{n, k}(\eta) .
$$

Note that:

$$
\begin{aligned}
\frac{\left(\alpha_{1}+n k\right)\left(\beta_{1}+n k\right)}{\left(\delta_{1}+n k\right)} & =n k+\left(\alpha_{1}+\beta_{1}-\delta_{1}\right)+\frac{\left(\delta_{1}-\alpha_{1}\right)\left(\delta_{1}-\beta_{1}\right)}{\left(\delta_{1}+n k\right)}, \\
\frac{\left(\delta_{2}+n k\right)\left(\beta_{2}+n k\right)}{\left(\delta_{2}+n k\right)} & =n k+\left(\alpha_{2}+\beta_{2}-\delta_{2}\right)+\frac{\left(\delta_{2}-\alpha_{2}\right)\left(\delta_{2}-\beta_{2}\right)}{\left(\delta_{2}+n k\right)}, \\
\frac{n k}{\delta_{1}+n k} & =1-\frac{\delta_{1}}{\delta_{1}+n k}, \quad \frac{n k}{\delta_{2}+n k}=1-\frac{\delta_{2}}{\delta_{2}+n k}
\end{aligned}
$$

and:

$$
\frac{1}{\left(\delta_{1}+n k\right)\left(\delta_{2}+n k\right)}=\frac{1}{\left(\delta_{1}-\delta_{2}\right)\left(\delta_{2}+n k\right)}-\frac{1}{\left(\delta_{1}-\delta_{2}\right)\left(\delta_{1}+n k\right)} .
$$


Using above the relations in (42), we obtain:

$$
\begin{aligned}
k^{2} \Theta^{2} \mathcal{H}(k ; \eta) & =k^{2} \eta\left\{\sum_{n=0}^{\infty} n^{2} k^{2} \mathbf{U}_{n, k}(\eta)+\left(\alpha_{1}+\beta_{1}+\alpha_{2}+\beta_{2}+\delta_{1}+\delta_{2}\right) \sum_{n=0}^{\infty} n k \mathbf{U}_{n, k}(\eta)\right. \\
& +\left(\delta_{1}-\alpha_{1}\right)\left(\delta_{2}-\beta_{1}\right)\left[\left(\alpha_{2}+\beta_{2}-\delta_{2}\right)-\delta_{1}-\frac{\left(\delta_{2}-\alpha_{2}\right)\left(\delta_{2}-\beta_{2}\right)}{\delta_{1}-\delta_{2}}\right] \sum_{n=0}^{\infty} \frac{1}{\delta_{1}+n k} \mathbf{U}_{n, k}(\eta) \\
& \left.\left(\delta_{2}-\alpha_{2}\right)\left(\delta_{2}-\beta_{2}\right)\left[\left(\alpha_{1}+\beta_{1}-\delta_{1}\right)-\delta_{2}+\frac{\left(\delta_{1}-\alpha_{1}\right)\left(\delta_{1}-\beta_{1}\right)}{\delta_{1}-\delta_{2}}\right] \sum_{n=0}^{\infty} \frac{\mathbf{U}_{n}(\eta)}{\delta_{2}+n k}\right\} \\
& =k^{2} \eta \Theta^{2} \mathcal{H}(k ; \eta)+\left(\alpha_{1}+\beta_{1}+\alpha_{2}+\beta_{2}-\delta_{1}-\delta_{2}\right) \eta \Theta k \mathcal{H}(k ; \eta) \\
& +\left[\left(\alpha_{1}+\beta_{1}-\delta_{1}\right)\left(\alpha_{2}+\beta_{2}-\delta_{2}\right)+\left(\delta_{1}-\alpha_{1}\right)\left(\delta_{1}-\beta_{1}\right)+\left(\delta_{2}-\alpha_{2}\right)\left(\delta_{2}-\beta_{2}\right)\right] \mathcal{H}(k ; \eta) \\
& +\left(\delta_{1}-\alpha_{1}\right)\left(\delta_{1}-\beta_{1}\right)\left[\left(\alpha_{2}+\beta_{2}-\delta_{2}\right)-\delta_{1}-\frac{\left(\delta_{2}-\alpha_{2}\right)\left(\delta_{2}-\beta_{2}\right)}{\delta_{1}-\delta_{2}}\right] \mathcal{H}\left(k ; \delta_{1}+\right) \\
& +\left(\delta_{2}-\alpha_{2}\right)\left(\delta_{2}-\beta_{2}\right)\left[\left(\alpha_{1}+\beta_{1}-\delta_{1}\right)-\delta_{2}+\frac{\left(\delta_{1}-\alpha_{1}\right)\left(\delta_{1}-\beta_{1}\right)}{\delta_{1}-\delta_{2}}\right] \mathcal{H}\left(k ; \delta_{2}+\right) .
\end{aligned}
$$

Thus, we get the following differential equation:

$$
\begin{aligned}
& {\left[(1-\eta) k^{2} \Theta^{2}-\left(\alpha_{1}+\beta_{1}+\alpha_{2}+\beta_{2}-\delta_{1}-\delta_{2}\right) \eta k \Theta\right.} \\
& \left.-\left\{\left(\alpha_{1}+\beta_{1}-\delta_{1}\right)\left(\alpha_{2}+\beta_{2}-\delta_{2}\right)+\left(\delta_{1}-\alpha_{1}\right)\left(\delta_{1}-\beta_{1}\right)+\left(\delta_{2}-\alpha_{2}\right)\left(\delta_{2}-\beta_{2}\right)\right\}\right] \mathcal{H}(k, \eta) \\
& -\left(\delta_{1}-\alpha_{1}\right)\left(\delta_{1}-\beta_{1}\right)\left[\left(\alpha_{2}+\beta_{2}-\delta_{2}\right)-\delta_{1} \frac{\left(\delta_{2}-\alpha_{2}\right)\left(\delta_{2}-\beta_{2}\right)}{\delta_{1}-\delta_{2}}\right] \mathcal{H}\left(k ; \delta_{1}+\right) \\
& -\left(\delta_{2}-\alpha_{2}\right)\left(\delta_{2}-\beta_{2}\right)\left[\left(\alpha_{1}+\beta_{1}-\delta_{1}\right)-\delta_{2}+\frac{\left(\delta_{1}-\alpha_{1}\right)\left(\delta_{2}-\beta_{1}\right)}{\delta_{1}-\delta_{2}}\right] \mathcal{H}\left(k ; \delta_{2}+\right)=0 .
\end{aligned}
$$

Remark 12. Taking $k=1$ in the above differential equation, it obviously reduces to the result of the generalized hypergeometric function in [32].

Yet another differential equation is satisfied by $\mathcal{H}(k ; \eta)$, as follows:

$$
\begin{aligned}
& {\left[k^{2} \Theta^{2}\left(\Theta+\delta_{1}-k\right)\left(\Theta+\delta_{2}-k\right)\right] \mathcal{H}(k, \eta)} \\
& =\sum_{n=0}^{\infty} \frac{k^{2} n^{2}\left(n k+\delta_{1}-k\right)\left(n k+\delta_{2}-k\right)}{\left(\delta_{1}\right)_{n, k}\left(\delta_{2}\right)_{n, k} n ! n !}\left(\alpha_{1}\right)_{n, k}\left(\alpha_{2}\right)_{n, k}\left(\beta_{1}\right)_{n, k}\left(\beta_{2}\right)_{n, k} \eta^{n} \\
& =\sum_{n=1}^{\infty} \frac{k^{2}\left(\alpha_{1}\right)_{n, k}\left(\alpha_{2}\right)_{n, k}\left(\beta_{1}\right)_{n, k}\left(\beta_{2}\right)_{n, k}}{\left(\delta_{1}\right)_{n-1, k}\left(\delta_{2}\right)_{n-1, k}(n-1) !(n-1) !} \eta^{n} \\
& =\sum_{n=0}^{\infty} \frac{k^{2}\left(\alpha_{1}\right)_{n+1, k}\left(\beta_{1}\right)_{n+1, k}\left(\alpha_{2}\right)_{n+1, k}\left(\beta_{2}\right)_{n+1, k}}{\left(\delta_{1}\right)_{n, k}\left(\delta_{2}\right)_{n, k} n ! n !} \eta^{n+1} \\
& =\sum_{n=0}^{\infty} \frac{k^{2}\left(\alpha_{1}\right)_{n+1, k}\left(\alpha_{2}\right)_{n+1, k}\left(\beta_{1}\right)_{n+1, k}\left(\beta_{2}\right)_{n+1, k}}{\left(\delta_{1}\right)_{n, k}\left(\delta_{2}\right)_{n, k} n ! n !} \eta^{n} \\
& =k^{2} \eta \sum_{n=0}^{\infty}\left(\alpha_{1}+n k\right)\left(\beta_{1}+n k\right)\left(\alpha_{2}+n k\right)\left(\beta_{2}+n k\right) \mathbf{U}_{n, k}(\eta) \\
& =k^{2} \eta\left(\Theta+\alpha_{1}\right)+\left(\Theta+\beta_{1}\right)+\left(\Theta+\alpha_{1}\right)\left(\Theta+\beta_{2}\right) \mathcal{H}(k, \eta) .
\end{aligned}
$$

Thus, we show that the $H(k, \eta)$ is a solution of the following differential equation:

$$
\left[k^{2} \Theta^{2}\left(\Theta+\delta_{1}-k\right)\left(\Theta+\delta_{2}-k\right)-\eta\left(\Theta+\alpha_{1}\right)\left(\Theta+\beta_{1}\right)\left(\Theta+\alpha_{2}\right)\left(\Theta+\beta_{2}\right)\right] \mathcal{H}(k, \eta)=0 .
$$

Remark 13. If we take $k=1$ in (42), we can easily obtain the known results given in [32]. 
Remark 14. The differential equation in (42) improves and generalizes the known results in [19,27].

\section{Concluding Remarks}

In this manuscript, we established a new generalization of the k-Gauss hypergeometric function by means of a Hadamard product, the so-called Hadamard product of k-Gauss hypergeometric functions (HPkGHF) of a fourth numerator and two denominator parameters. We presented a systematic study of the various basic properties of the HP$\mathrm{kGHF}$, including the convergence property, differentiation formulae, integral transforms, contiguous function relations, and differential equations. The investigated results presented here along with those presented in earlier papers were specified. Moreover, some interesting special cases of our main results were considered at $k \rightarrow 1$.

Furthermore, it is noted that the results detailed in this article are general in character and give some contributions to the theory of special functions. In addition, the results proposed in this work can be easily converted in terms of a similar type of new interesting integral formulae and differential equations with different arguments after some suitable parameter replacements.

More importantly, We are also trying to find certain possible applications of the results reported here to some other research areas, such as random walk, distributed delay, fractional operators, numerical solutions, and boundary value problems (see, [35-42]).

Author Contributions: Writing-original draft, M.A., M.H.; Writing-review and editing, M.A., M.H. All authors have read and agreed to the published version of the manuscript.'

Funding: The first named author extends their appreciation to the Deanship of Scientific Research at King Khalid University for funding this work through the Research Groups Program under Grant (R.G.P.-1/3/42).

Institutional Review Board Statement: Not applicable.

Informed Consent Statement: Not applicable.

Data Availability Statement: Not applicable.

Conflicts of Interest: The authors declare no conflict of interest.

\section{References}

1. Agarwal, P.; Agarwal, R.; Ruzhansky, M. Special Functions and Analysis of Differential Equations, 1st ed.; CRC Press: Boca Raton, FL, USA, 2020.

2. Akhmedova, V.; Akhmedov, E. Selected Special Functions for Fundamental Physics; SpringerBriefs in Physics; Springer: Cham, Switzerland, 2019.

3. Seaborn, J. Hypergeometric Functions and Their Applications; Springer: New York, NY, USA, 1991.

4. Sneddon, I. Special Functions of Mathematical Physics and Chemistry; Oliver and Boyd: Edinburgh, UK, 1956.

5. Srivastava, H.; Rahman, G.; Nisar, K. Some extensions of the Pochhammer Symbol and the associated hypergeometric functions. Iran. J. Sci. Technol. Trans. Sci. 2019, 43, 2601-2606. [CrossRef]

6. Srivastava, H.; Tassaddiq, A.; Rahman, G.; Nisar, K.; Khan, I. A new extension of the t-Gauss hypergeometric function and its associated properties. Mathematics 2019, 7, 996. [CrossRef]

7. Jana, R.; Maheshwari, B.; Shukla, A.K. Note on extended hypergeometric function. Proyecciones (Antofagasta) 2019, 38, 585-595. [CrossRef]

8. Jana, R.; Maheshwari, B.; Shukla, A. Some results on the extended hypergeometric function. J. Indian Math. Soc. 2020, 87, 70. [CrossRef]

9. Goswami, A.; Jain, S.; Agarwal, P.; Araci, S. A note on the new extended beta and Gauss hypergeometric functions. Appl. Math. Inf. Sci. 2018, 12, 139-144. [CrossRef]

10. Agarwal, P.; Choi, J.; Jain, S. Extended hypergeometric functions of two and three variables. Commun. Korean Math. Soc. 2015, 30, 403-414. [CrossRef]

11. Fuli, H.; Bakhet, A.; Hidan, M.; Abdalla, M. On the extended hypergeometric matrix functions and their applications for the derivatives of the extended Jacobi matrix polynomial. Math. Eng. 2020, 2020, 4268361.

12. Hidan, M.; Boulaaras, S.; Cherif, B.; Abdalla, M. Further results on the $(p ; k)$ analogue of hypergeometric functions associated with fractional calculus operators. Math. Probl. Eng. 2021, 2021, 5535962. [CrossRef]

13. Hidan, M.; Abdalla, M. A note on the Appell hypergeometric matrix function $F_{2}$. Math. Probl. Eng. 2020, 2020, 6058987. [CrossRef] 
14. Abdalla, M. Special matrix functions: Characteristics, achievements and future directions. Linear Multilinear Algebra 2020, 68, 1-28. [CrossRef]

15. Abdalla, M. Fractional operators for the Wright hypergeometric matrix functions. Adv. Differ. Equ. 2020, 2020, 246. [CrossRef]

16. Abdalla, M. Further results on the generalized hypergeometric matrix functions. Int. J. Comput. Sci. Math. 2019, 10, 1-10. [CrossRef]

17. Abdalla, M.; Bakhet, A. Extended Gauss hypergeometric matrix functions. Iran. J. Sci. Technol. Trans. Sci. 2018, 42, 1465-1470. [CrossRef]

18. Diaz, R.; Pariguan, E. On hypergeometric functions and k- Pochhammer symbol. Divulg. Mat. 2007, 15, 179-192.

19. Mubeen, S.; Naz, M.; Rehman, A.; Rahman, G. Solutions of k-hypergeometric differential equations. J. Appl. Math. 2014, 2014, 13. [CrossRef]

20. Mubeen, S.; Rahman, G.; Rehman, A.; Naz, M. Contiguous function relations for k-hypergeometric Functions. ISRN Math. Anal. 2014, 2014, 410801. [CrossRef]

21. Mubeen, S.; Habibullah, G. An integral representation of some k-hypergeometric functions. Int. Math. Forum. $2012,7,203-207$.

22. Mubeen, S. k-Analogue of Kummers first formula. J. Inequal. Spec. Funct. 2012, 3, 41-44.

23. Rahman, G.; Mubeen, S.; Nisar, K. On generalized k- fractional derivative operator. AIMS Math. 2020, 5, 1936-1945. [CrossRef]

24. Chinra, S.; Kamalappan, V.; Rakha, M.; Rathie, A. On several new contiguous function relations for k-hypergeometric function with two parameters. Commun. Korean Math. Soc. 2017, 32, 637-651.

25. Korkmaz-Duzgun, D.; Erkus-Duman, E. Generating functions for k-hypergeometric functions. Int. J. Appl. Phys. Math. 2019, 9, 119-126.

26. Nisar, K.; Qi, F.; Rahman, G.; Mubeen, S.; Arshad, M. Some inequalities involving the extended gamma function and the Kummer confluent hypergeometric k-function. J. Inequal. Appl. 2018, 2018, 135. [CrossRef] [PubMed]

27. Li, S.; Dong, Y. k-hypergeometric series solutions to one type of non-homogeneous k-hypergeometric equations. Symmetry 2019, 11, 262. [CrossRef]

28. Kiryakova, V. Unified approach to fractional calculus images of special functions-A survey. Mathematics 2020, 8, 2260. [CrossRef]

29. Yilmazer, R.; Ali, K. Discrete fractional solutions to the k-hypergeometric differential equation. Math. Meth. Appl. Sci. 2020, 18. [CrossRef]

30. Sadykov, T. The Hadamard product of hypergeometric series. Bull. Sci. Math. 2002, 126, 31-43. [CrossRef]

31. Jain, S.; Nieto, J.; Singh, G.; Choi, J. Certain generating relations involving the generalized multi-index Bessel-Maitland and function. Math. Probl. Eng. 2020, 2020, 8596736. [CrossRef]

32. Rainville, E. Special Functions; The Macmillan: New York, NY, USA, 1960.

33. Milovanovi, G.; Parmar, R.; Rathie, A. A Study Of generalized summation theorems for the series ${ }_{2} F_{1}$ with an applications to laplace transforms of convolution type integrals involving Kummer's functions ${ }_{1} F_{1}$. Appl. Anal. Discret. Math. 2018, 12, $257-272$. [CrossRef]

34. Koepf, W.; Kim, I.; Rathie, A. On a new class of Laplace-type integrals involving generalized hypergeometric functions. Axioms 2019, 8, 87. [CrossRef]

35. Byteva, V.; Kalmykovb, M.; Olafmoch, S. Hypergeometric functions differential reduction (hyperdire): Mathematic Abased packages for differential reduction of generalized hypergeometric functions: FD and FS Horn-type hypergeometric functions of three variables. Comput. Phys. Commun. 2014, 185, 3041-3058. [CrossRef]

36. Guefaifia, R.; Boulaaras, S.M.; El-Sayed, A.A.; Abdalla, M.; Cherif, B. On existence of sequences of weak solutions of fractional systems with Lipschitz nonlinearity. J. Funct. Spaces 2021, 2021, 5510387.

37. Kamache, F.; Boulaaras, S.M.; Guefaifia, R.; Chung, N.T.; Cherif, .; Abdalla, M. On existence of multiplicity of weak solutions for a new class of nonlinear fractional boundary value systems via variational approach. J. Funct. Spaces 2021, 2021, 5544740.

38. Kaiblinger, N. Product of two hypergeometric functions with power arguments. J. Math. Anal. Appl. 2019, 479, 2236-2255 [CrossRef]

39. Boulaaras, S.; Choucha, A.; Cherif, B.; Alharbi, A.; Abdalla, M. Blow up of solutions for a system of two singular nonlocal viscoelastic equations with damping, general source terms and a wide class of relaxation functions. AIMS Math. 2021, 6, 4664-4676. [CrossRef]

40. Ouchenane, D.; Choucha, A.; Boulaaras, M.A.S.; Cherif, B. On the Porous-Elastic System with Thermoelasticity of Type III and Distributed Delay: Well-Posedness and Stability. J. Funct. Spaces 2021, 2021, 9948143.

41. Choucha, A.; Boulaaras, S.; Ouchenane, D.; Alkhalaf, S.; Mekawy, I.; Abdalla, M. On the system of coupled nonde generate Kirchhoff equations with distributed delay: Global existence and exponential decay. J. Funct. Spaces 2021, $2021,5577277$.

42. Naz, S.; Naeem, M.N. On the generalization of k-fractional Hilfer-Katugampola derivative with Cauchy problem. Turk. J. Math. 2021, 45, 110-124. [CrossRef] 